\section{Cuidado pós-parto às mulheres na atenção primária: construção de um modelo avaliativo}

\author{
Postpartum care for women in primary care: \\ building an assessment model
}

\section{Cuidado posparto a mujeres en la atención primaria: construcción de un modelo de evaluación}

Tatiane Baratieri 1,2

Sonia Natal 2

Zulmira Maria de Araújo Hartz 3

\section{Resumo}

A assistência pós-parto às mulheres na atenção primária à saúde (APS) é importante para a redução da morbimortalidade destas, porém não há uma teoria desta assistência claramente descrita e os estudos avaliativos são escassos. Este trabalho objetivou desenvolver e sistematizar um modelo avaliativo da assistência às mulheres no pós-parto na APS, verificando sua avaliabilidade. Realizou-se um Estudo de Avaliabilidade por meio de revisão da literatura nacional e internacional, revisão de documentos nacionais e entrevistas com stakeholders. Tais evidências fundamentaram a elaboração do modelo avaliativo que foi validado em conferência de consenso. Utilizou-se análise temática para a análise dos dados. O estudo elaborou a teoria do programa, em que a assistência pós-parto na APS precisa idealmente ocorrer de forma integral, conforme as necessidades de saúde física, psicológica, emocional e social; considerar a individualidade das mulheres com filhos vivos ou em situações de óbito fetal/infantil; iniciar no pré-natal e ter continuidade no pós-parto, com envolvimento das famílias/companheiros(as). A teoria do programa estabeleceu a contextualização e um desenho lógico com objetivo, metas, atividades, produtos, resultados e impacto, até então não sistematicamente explicitados na literatura e documentos. Verificou-se que a assistência pós-parto é avaliável por meio de uma análise de implantação e que a teoria do programa definida pode ser utilizada por diversos atores, tanto em nível nacional quanto internacional, para implantar e/ou aprimorar a assistência integral às mulheres no pós-parto.

Período Pós-Parto; Avaliação em Saúde; Saúde das Mulheres; Atenção Primária à Saúde

\section{Correspondência}

T. Baratieri

Departamento de Enfermagem, Universidade Estadual do Centro-Oeste.

Al. Élio Antonio Dalla Vecchia 838, Guarapuava, PR 85040-167, Brasil.

baratieri.tatiane@gmail.com

1 Universidade Estadual do Centro-Oeste, Guarapuava, Brasil. 2 Universidade Federal de Santa Catarina, Florianópolis, Brasil. 3 Universidade NOVA de Lisboa, Lisboa, Portugal. 


\section{Introdução}

O pós-parto é um período de intensas mudanças físicas, psicológicas e sociais para as mulheres, no qual a maioria delas sofre algum tipo de intercorrência, necessitando de auxílio da rede de apoio - serviços de saúde, comunidade, familiares, entre outros - para superar os problemas 1 . Assim, é fundamental uma assistência à saúde de qualidade, considerando que tais mudanças podem levar a agravos, resultando em morbidades temporárias ou permanentes, e mesmo em mortes 1,2,3, sendo esta uma temática que tem feito parte da agenda internacional de políticas públicas.

Os cuidados às mulheres no pós-parto podem ser definidos como práticas e avaliações de cuidados preventivos de rotina que são planejados para identificar, gerenciar ou encaminhar complicações maternas 4 . Tais cuidados, quando prestados por profissionais no âmbito da atenção primária à saúde (APS), impactam positivamente na saúde e qualidade de vida das mulheres, com redução da morbimortalidade, aumento da satisfação destas com o cuidado e melhora na autonomia das mesmas 5,6,7,8,9.

Para a garantia de bons resultados à saúde das mulheres, faz-se necessário que a assistência pós-parto seja organizada de forma coerente e aplicável, com foco nas suas necessidades de saúde, proporcionando atenção integral e superação da visão das mulheres como reprodutoras, arraigada nas políticas de saúde nacionais e internacionais 6,10,11.

A identificação da adequada operaracionalização de uma política ou programa de saúde pode ser realizada por meio de uma avaliação, mas previamente ao processo avaliativo propriamente dito, é necessário conhecer a lógica do programa, assim como a teoria envolvida na sua elaboração 12 .

Atualmente, estudos avaliativos da atenção pós-parto são escassos, e os existentes, em geral, têm foco normativo ou em programas específicos do período pós-parto, como programas de assistência à depressão pós-parto ou programas de visita domiciliar 13, ou seja, tais estudos não estabelecem a operacionalização do programa pós-parto e os aspectos que o envolvem para então proceder a avaliação.

Para suprir essas necessidades, o Estudo de Avaliabilidade tem sido usado no campo da avaliação em saúde, com crescimento nos últimos anos, tanto nacional como internacionalmente, por ser uma abordagem sistemática para o planejamento de projetos de avaliação, e tem se mostrado útil para melhorias de programas 14,15 .

Esse tipo de estudo pode envolver a realização de uma pré-avaliação para determinar se um programa está pronto para ser avaliado, indicar aspectos para melhorias, aprimorar o conhecimento sobre o programa, garantir que uma avaliação produza informações úteis, envolver os interessados na avaliação, esclarecer as metas de intervenção e como elas devem ser alcançadas, e elaborar, testar e refinar uma teoria de mudança acordada com interessados 15,16,17, podendo ser utilizado em qualquer fase do programa, proporcionando aprofundamento no conhecimento sobre o funcionamento do mesmo 16,17 .

Assim, a necessidade de realizar um Estudo de Avaliabilidade da assistência pós-parto justifica-se por: escassez de trabalhos que avaliem a assistência às mulheres no pós-parto para além de aspectos normativos ou de "recortes" do programa 13; ser uma assistência ainda negligenciada pelas políticas públicas, serviços e profissionais de saúde 7; elevada morbimortalidade das mulheres no período pós-parto 4,6,7,10; pela necessidade de conhecer a operacionalização da assistência pós-parto considerando a atenção integral às mulheres, superando o materno-infantilismo 6,10,11,13.

Frente ao exposto, objetivou-se desenvolver e sistematizar um modelo avaliativo da assistência às mulheres no pós-parto na APS, verificando a avaliabilidade.

\section{Método}

Trata-se de um Estudo de Avaliabilidade de abordagem qualitativa, desenvolvido de novembro de 2016 a dezembro de 2018, seguindo os pressupostos de Thurston \& Ramaliu 18, Leviton et al. 17, e Trevisan \& Walser 16, teóricos amplamente utilizados na área da saúde e atendendo o rigor metodológico para este tipo de trabalho 14,19 . 
Para a operacionalização do estudo realizou-se uma revisão da literatura ${ }^{16}$, análise documental 16,17,18 e entrevista com stakeholders (interessados na avaliação), como parte do processo de envolvimento dos mesmos 16,17,18. Esses, participaram em todo o Estudo de Avaliabilidade, desde a concepção da necessidade de avaliação até a validação final do modelo avaliativo.

A revisão da literatura ocorreu por meio de uma revisão integrativa e uma revisão sistemática. A integrativa, realizada de novembro de 2016 a março de 2017, abordou as ações de programas de atenção pós-parto no âmbito da APS, tanto em nível nacional como internacional 13. A sistemática foi realizada entre os meses de abril de 2017 e fevereiro de 2018, e tratou de recomendações de diretrizes clínicas internacionais sobre a atenção às mulheres no pós-parto na APS 20.

Para a análise documental 16,17,18, ocorrida de março a julho de 2018, foram usados cinco documentos nacionais 11,21,22,23,24 vigentes, que tratam da atenção pós-parto na APS, localizados na página da Internet do Ministério da Saúde (http://saude.gov.br/).

Quanto aos stakeholders, realizou-se entrevista semiestruturada, de agosto a outubro de 2018, com quatro mulheres representantes de movimento de mulheres, cinco profissionais da área de APS e saúde das mulheres da gestão dos estados do Paraná, Santa Catarina e Rio Grande do Sul e três profissionais com experiência na APS. Os participantes foram selecionados por sua reconhecida experiência acadêmica e/ou profissional na Região Sul do país na área de saúde das mulheres, avaliação em saúde e/ou APS. As entrevistas foram realizadas em sala reservada, gravadas e transcritas na íntegra.

Para a coleta dos dados na revisão da literatura, análise documental e entrevista foram utilizadas as questões-chave propostas por Bezerra et al. ${ }^{25}$, que auxiliam o delineamento de um programa (Quadro 1).

\section{Quadro 1}

Questões-chave para a construção do modelo avaliativo da assistência às mulheres no pós-parto na atenção primária à saúde (APS) e respectiva fonte de evidência. Brasil, 2018.

\begin{tabular}{|c|c|c|c|c|c|c|c|}
\hline \multirow[t]{2}{*}{ QUESTÕES-CHAVE } & \multicolumn{7}{|c|}{ FONTES DE EVIDÊNCIAS } \\
\hline & F1 & $\mathbf{F 2}$ & F3 & F4 & F5 & F6 & F7 \\
\hline 1) Qual o problema visado pelo programa, pelos seus formuladores, executores ou agentes locais? & $\mathrm{X}$ & $\mathrm{X}$ & X & $\mathrm{X}$ & $X$ & & $\mathrm{X}$ \\
\hline 2) Qual o programa de saúde criado para resolver o problema? & $\mathrm{X}$ & $\mathrm{X}$ & X & $x$ & $x$ & & $x$ \\
\hline 3) Qual o objetivo geral do programa? & $\mathrm{X}$ & $\mathrm{X}$ & $x$ & & $x$ & & $x$ \\
\hline 4) Quais são os seus objetivos específicos? & & $\mathrm{X}$ & & $\mathrm{X}$ & & & $x$ \\
\hline 5) Quais as metas que o programa pretende alcançar? & $\mathrm{X}$ & & & & & & $x$ \\
\hline 6) Qual é a sua população-alvo? & $\mathrm{X}$ & $\mathrm{X}$ & X & $x$ & $x$ & & $x$ \\
\hline 7) Quais são os componentes do programa? & $\mathrm{X}$ & & & & $x$ & & $x$ \\
\hline 8) Que atividades são realizadas? & $\mathrm{X}$ & $\mathrm{X}$ & $\mathrm{X}$ & $\mathrm{X}$ & $\mathrm{X}$ & $\mathrm{X}$ & $\mathrm{X}$ \\
\hline 9) Quais as estruturas que o programa precisa para funcionar? & $\mathrm{X}$ & & & & $\mathrm{X}$ & & $X$ \\
\hline 10) Quais os produtos que se espera obter com a realização das atividades? & & $\mathrm{X}$ & & & & & $\mathrm{X}$ \\
\hline 11) Quais os resultados que o programa pretende alcançar? & & & & & & & $\mathrm{x}$ \\
\hline 12) Quais os fatores que podem influenciar no alcance dos resultados, além dos relacionados ao programa? & $\mathrm{X}$ & $\mathrm{X}$ & & & & & $\mathrm{X}$ \\
\hline
\end{tabular}

F1: revisão integrativa 13; F2: revisão sistemática 20; F3: Protocolos de Atenção Básica: Saúde das Mulheres 11; F4: Atenção ao Pré-Natal de Baixo Risco (Cadernos de Atenção Básica, 32) 22; F5: regulamentação da Rede Cegonha 21,24; F6: Cadernos HumanizaSUS: Humanização do Parto e do Nascimento 23; F7: entrevista com stakeholders. 
Com base na revisão da literatura, análise documental e entrevista com stakeholders foram definidos os objetivos e metas da assistência pós-parto 17,18 e realizada a modelização inicial do programa, ou seja, a elaboração do Modelo Lógico do Programa (MLP) e do Modelo Teórico de Avaliação (MTA) $16,17,18$.

Para a validação dos objetivos/metas, modelização do programa 17,18, pactuação da pergunta avaliativa e dos procedimentos de avaliação possíveis para o presente objeto 17 , foi utilizada a técnica de conferência de consenso 17,18 junto a especialistas da área e stakeholders, de acordo com a metodologia de Conferência de Consenso proposta por Souza et al. 26. Participaram da Conferência de Consenso duas representantes de organizações de mulheres, dois profissionais da APS, cinco profissionais da área de APS e saúde das mulheres da gestão dos estados do Paraná, Santa Catarina e Rio Grande do Sul, e cinco especialistas em avaliação em saúde das mulheres.

Após a validação do modelo avaliativo foram elaboradas as recomendações para o uso do Estudo de Avaliabilidade 16,17.

A análise temática 27 foi aplicada para a análise do material da revisão da literatura, documentos e dados das entrevistas com stakeholders, de acordo com uma lista pré-definida de códigos proposta pelas autoras, buscando identificar os objetivos e metas, a operacionalização e os contextos que influenciam o programa. O software de análise NVivo versão 11 (https://www.qsrinternational.com/ nvivo/home) foi usado para facilitar a codificação dos dados das entrevistas.

O trabalho foi aprovado pelo Comitê de Ética em Pesquisa com Seres Humanos da Universidade Federal de Santa Catarina (parecer no 2.814.988/2018).

\section{Resultados}

O presente modelo avaliativo tem como premissa a prestação do cuidado integral às mulheres no pós-parto, incluindo as mulheres com filhos nascidos vivos ou óbito fetal/infantil, pelo período e frequência de atendimento que as mesmas necessitarem. Os resultados do Estudo de Avaliabilidade foram apresentados em três eixos temáticos sobre o modelo avaliativo e um tópico sobre o uso do Estudo de Avaliabilidade.

\section{Identificando o objetivo e as metas}

Verificou-se que apenas uma diretriz clínica, dentre as analisadas na revisão sistemática 20, trouxe de forma clara o objetivo da assistência pós-parto na APS, entendido como fornecer consistência de aconselhamento e apoio para a recuperação da gravidez e do nascimento, identificação precoce e gestão adequada das necessidades de saúde física e psicológica, emocional e social, e facilitar o início da vida familiar 6,28. As demais fontes de evidências apresentaram o objetivo de forma implícita no decorrer do material 7,10, corroborando com o documento do Instituto Nacional pela Excelência do Cuidado e da Saúde do Reino Unido (NICE) 6,28, e acrescentaram questões como prestar atenção integral 11,13 e humanizada 21,24.

Os stakeholders contribuíram para ratificar os objetivos encontrados na literatura/documentos, principalmente quanto à atenção integral, recuperação do parto, orientações para aumentar a autonomia das mulheres, e incluíram a questão do envolvimento da família/companheiros(as):

"É ter garantida sua saúde, sua felicidade, seu bem-estar dentro das propostas de atenção integral..." (Stakeholder 3).

"Fazer com essa mulher uma conversa no sentido de como que foi o parto, pra pessoa superar aquilo, para dar os próximos passos" (Stakeholder 1).

"Acompanhar, orientar, mesmo que esteja bem, para ter conhecimento, empoderamento. Muitas vezes a mulher não sabe o que é normal, não sabe o que ela precisa ter cuidado" (Stakeholder 9).

“...O companheiro(a) e família precisam ouvir as orientações também, pois a mulher sozinha não consegue mudar sua realidade, não consegue protagonizar mudanças" (Stakeholder 2).

Com base no exposto, o objetivo pactuado junto com stakeholders e especialistas em Conferência de Consenso foi: "prestar atenção integral por meio do aconselhamento e apoio para a recuperação da gravidez, 
parto e nascimento, continuidade do cuidado pré-natal, identificação oportuna de situações de risco e vulnerabilidade, e gestão das necessidades de saúde física, psicológica, emocional e social das mulheres, em conjunto com a família e/ou companheiros(as), além de facilitar a adaptação em família”.

Além disso, emergiu dos stakeholders a importância da assistência pós-parto para todas as mulheres, independentemente de terem filhos nascidos vivos ou não: "E não é só quando o bebê nasce vivo (...) $e$ quando morre? A mulher precisa de atenção, de cuidados, teve um parto do mesmo jeito e ainda com um problema que é a morte do bebê. Mas as pessoas não entendem, dão uma semana de folga e pronto, não tem bebê, então tudo volta ao normal, mas não é assim, a mulher precisa de apoio, precisa se recuperar do parto e precisa de cuidado por causa da depressão" (Stakeholder 10).

Os documentos nacionais e as diretrizes internacionais 20 não estabelecem metas para a assistência pós-parto, e os stakeholders não conseguiram defini-las claramente, por vezes as confundiram com o objetivo do programa. Assim, as metas foram estabelecidas: com base nas principais necessidades das mulheres no pós-parto, identificadas junto aos stakeholders e documentos/literatura; fundamentadas em estudos sobre cada tema abordado; ajustadas e validadas em conferência de consenso, sendo definidas como:

(i) Acompanhar e aconselhar 100\% das mulheres no pós-parto, junto com companheiros(as) e famílias 7,10,11;

(ii) Identificar no mínimo $80 \%$ de mulheres com sintomas físicos e/ou psicológicos, emocional e social e tratar/atender cada caso 2,29,30;

(iii) Identificar no mínimo 10\% de mulheres com sinais de violência doméstica no pós-parto e prestar assistência qualificada 31 ;

(iv) Identificar no mínimo $10 \%$ de mulheres com depressão pós-parto e prestar assistência qualificada 32;

(v) Reduzir para menos de $20 \%$ o número de mulheres com gravidez de repetição rápida 33;

(vi) Aumentar a taxa de aleitamento materno exclusivo para $50 \%$ em crianças de até 6 meses 34.

\section{Contextualizando o programa}

A contextualização do programa envolve a compreensão da teoria em que são identificados fatores de contexto que podem influenciar o mesmo, localização de onde a assistência pós-parto está inserida no Sistema Único de Saúde (SUS), suas relações e os atores envolvidos. Esse entendimento orienta o modo como o programa idealmente funciona e pode ser representado por meio do MTA (Figura 1).

Identificaram-se distintos fatores contextuais que podem atuar sobre a assistência pós-parto, os quais se referem ao contexto externo (fatores socioeconômicos/demográficos) e político-institucional do programa.

Sobre o contexto externo, verificou-se que as crenças da população relacionadas aos cuidados pós-parto exigem dos profissionais compreensão e adaptação do cuidado. As concepções arraigadas tanto da população como de profissionais e gestores quanto às desigualdades de gênero podem levar a assistência reducionista ao papel das mulheres como reprodutoras 7,10,11,13,28,35. As condições socioeconômicas e demográficas podem interferir na adesão às orientações e nas condições adequadas para os cuidados, considerando que, em geral, população de baixa renda tem acesso limitado aos bens de consumo e condições precárias de saneamento básico, moradia, entre outros 7 .

Os stakeholders contribuíram principalmente para reforçar questões de gênero presentes tanto na prática profissional quanto no convívio social das mulheres, e destacaram a influência das crenças da população sobre o pós-parto:

"A saúde da mulher no pós-parto é negligenciada, não superamos o materno-infantilismo. A atenção à mulher se encerra com o nascimento da criança" (Stakeholder 4).

"Os homens não têm maturidade, começa ter ciúme do filho, é uma loucura isso (...) e tem aquela ótica machista, que está na cabeça das mães, sogras, que ficam indignadas de ver o filho trocando fralda” (Stakeholder 3).

“...Tem bastante resistência do que pode, o que não pode ser feito, com relação à higienização da mulher, lavar o cabelo, a dieta alimentar, o resguardo" (Stakeholder 5).

Sobre o contexto político-institucional há necessidade da articulação entre as três esferas de governo 11,21,24. Os atributos da APS (acesso, longitudinalidade, integralidade e coordenação do 
Figura 1

Modelo Teórico de Avaliação da assistência às mulheres no pós-parto na atenção primária à saúde (APS). Brasil, 2018.

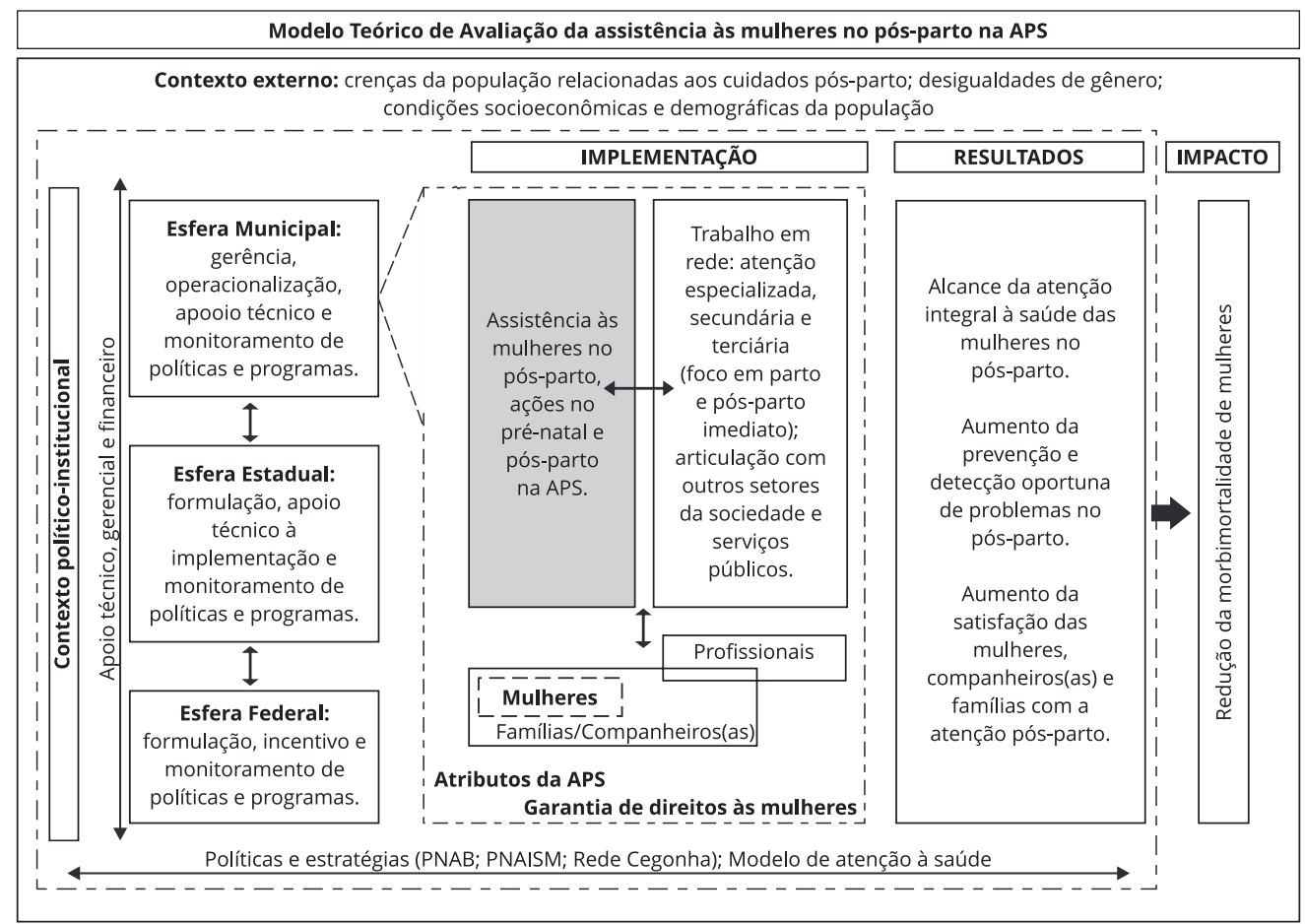

PNAB: Política Nacional de Atenção Básica; PNAISM: Política Nacional de Atenção Integral à Saúde das Mulheres.

cuidado), a garantia de direitos às mulheres, políticas e programas no âmbito da saúde das mulheres e APS, e a configuração do modelo de atenção à saúde são norteadores de todo o processo de assistência pós-parto 6,10,11,13,28,36.

Após parto, o cuidado às mulheres ocorre predominantemente na APS, que é a ordenadora do cuidado no âmbito da rede de atenção à saúde (RAS), com horizontalidade nas relações entre os pontos de atenção, além da atuação conjunta com outros setores públicos e da sociedade, objetivando oferta de atenção contínua e integral 7,10,11,13,21,24,28.

A assistência às mulheres para o pós-parto começa no pré-natal e deve ter continuidade durante o período pós-parto, com os profissionais atuando conforme as necessidades de saúde das mulheres e o envolvimento das famílias/companheiros(as) 7,10,11,13,28.

Sobre o contexto político-institucional, os conteúdos mais presentes nas falas dos stakeholders foram em relação ao papel das esferas de governo, a atenção integral, articulação intersetorial e trabalho em rede:

"O Ministério da Saúde tem que formular as políticas para as mulheres (...). A gestão estadual presta apoio técnico e financeiro, fomenta-se a construção de espaços coletivos para a gestão da política de saúde das mulheres, e o município tem que apoiar diretamente as equipes" (Stakeholder 4).

"A atenção integral deve orientar a assistência a essa mulher" (Stakeholder 10).

"Tem que ter o serviço de referência e contrarreferência e também organizar aquelas rodas de conversa, acompanhadas por assistentes social, psicóloga ou feministas" (Stakeholder 3). 


\section{Operacionalizando o programa}

Com a finalidade de demonstrar como o programa opera 12 , foi elaborado o MLP, representado graficamente pela Figura 2. O MLP é constituído por duas dimensões (Gestão e Execução), que têm subdimensões com atividades específicas, a fim de constituir a lógica do programa na ótica da assistência integral às mulheres no pós-parto.

\section{Figura 2}

Modelo Lógico do Programa da assistência às mulheres no pós-parto na atenção primária à saúde (APS). Brasil, 2018.

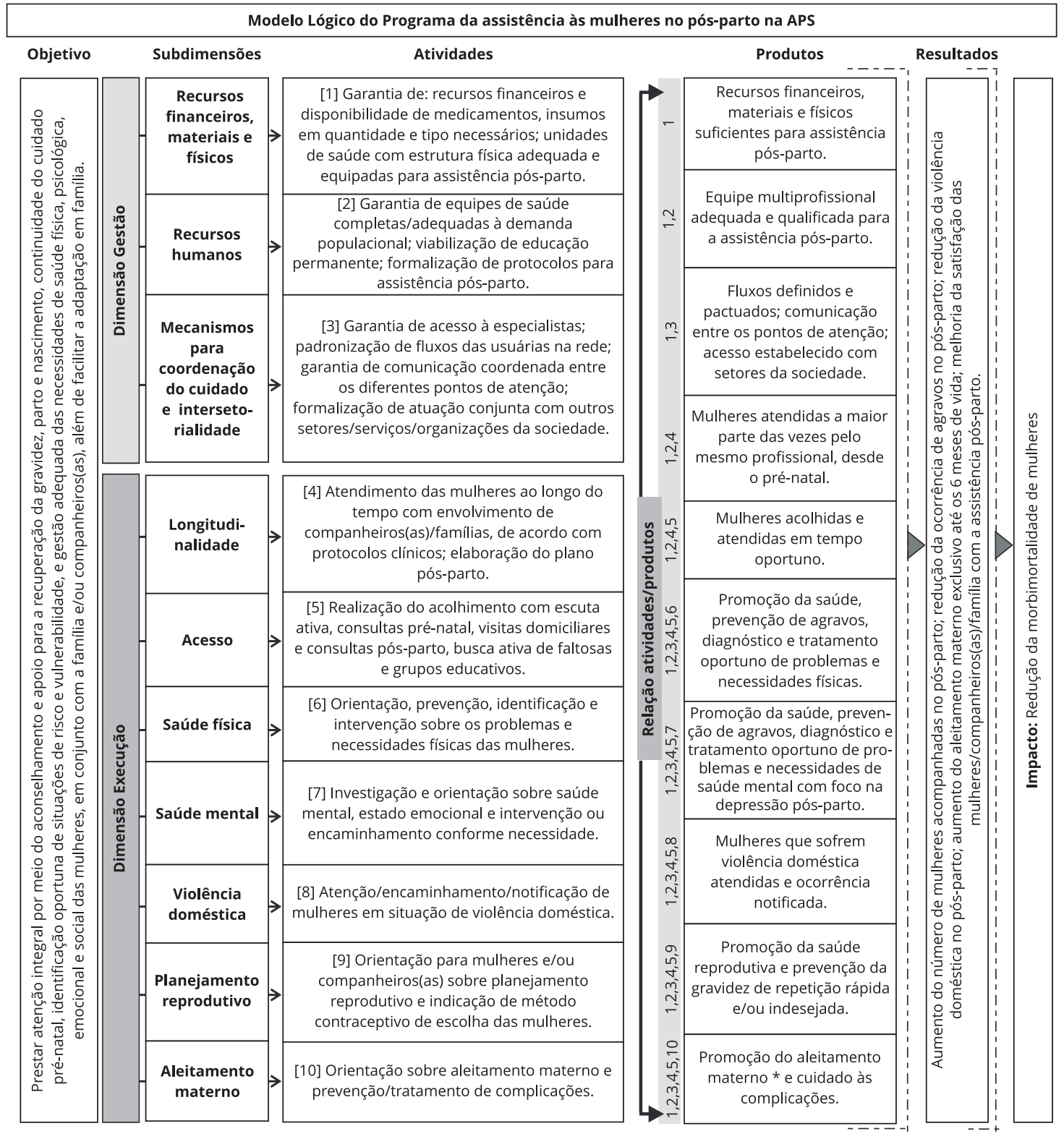

* Para mulheres com recém-nascido vivo. 
Com base nas atividades resultam produtos, os quais decorrem da execução de uma ou mais atividades no âmbito das diferentes dimensões e subdimensões. Das atividades e produtos provêm os resultados, que refletem o alcance das metas estabelecidas para a população-alvo. Finalmente, o MLP apresenta o impacto, que é caracterizado como o alcance das metas na população geral 11,13,20,21,22,24.

A dimensão Gestão busca identificar as atividades e responsabilidades no âmbito da gestão municipal de saúde quanto à provisão de recursos (financeiros, humanos, materiais e físicos), qualificação dos profissionais e da assistência, e garantia de articulação na RAS, tendo a APS como ordenadora do cuidado, com a finalidade de viabilizar a assistência pós-parto de qualidade 7,11,13,21,22,24.

Os stakeholders destacaram principalmente a importância da gestão municipal de saúde garantir recursos, qualificar profissionais e garantir trabalho intersetorial:

"O município tem que dar as condições para que a atenção básica atenda essas mulheres, os recursos necessários. E também tem que preparar os profissionais, capacitar eles” (Stakeholder 12).

"Instituir protocolos, que a gestão de cada município coloque isso como regra (assistência pós-parto), pra todas as unidades terem o mesmo padrão" (Stakeholder 6).

"A gestão tem que pensar junto com a assistência social em fortalecer a rede familiar. Tem que trabalhar na rede, se a mulher está num estado depressivo, precisando desse suporte, de repente também ser pensado nisso com os CRAS [Centro de Referência de Assistência Social]” (Stakeholder 1).

A dimensão Execução caracteriza-se pelo escopo de ações/atividades desenvolvidas pelos profissionais da APS a fim de prestar atenção integral, considerando as necessidades de saúde e escolhas das mulheres, com envolvimento de companheiros(as) e/ou família. A assistência deve iniciar durante o pré-natal com a elaboração do plano de cuidados pós-parto, e ter continuidade após o parto por meio de acesso oportuno e cuidado longitudinal, englobando ações quanto à saúde física, saúde mental, violência doméstica, planejamento reprodutivo e aleitamento materno 11,13,20,21,22,24.

Sobre a referida dimensão, os stakeholders reforçaram o disposto na literatura/documentos, com ênfase ao acompanhamento, saúde mental, saúde sexual e violência:

"Fazer a consulta pós-parto e a visita domiciliar. Eu não posso colocar em protocolo, você vai voltar daqui 15 dias, daqui um mês, não posso, porque vai depender do contexto dela" (Stakeholder 5).

“Tem a questão psicológica, da depressão pós-parto que a gente precisa estar atenta, precisa ser atendida, ser explicada” (Stakeholder 8).

"São poucos profissionais que trabalham a sexualidade. Ela vai estar insegura, vai ter alterações hormonais, ela tem que ser orientada, preparada" (Stakeholder 7).

"Trabalhar a violência sexual, por exemplo, a mulher acha que é normal ser pressionada pelo companheiro para manter relações sexuais” (Stakeholder 2).

\section{Propondo recomendações para o uso do Estudo de Avaliabilidade}

O Estudo de Avaliabilidade elaborou o desenho da intervenção que inclui objetivos, ações e metas não sistematizadas na literatura, em conjunto com todos os envolvidos no processo. Foi útil especialmente para os stakeholders, oportunizando aprimorar a compreensão a respeito do programa, o que pode ter estimulado a reflexão sobre este e lhes auxiliar a protagonizar mudanças em sua realidade.

Estabeleceu que é possível avaliar a assistência pós-parto na APS e orientou decisões sobre o processo avaliativo. Definiu-se, em conjunto com stakeholders e especialistas, que o melhor tipo de avaliação é o de implantação, a fim de identificar as ações realizadas, as potencialidades, lacunas e contextos que influenciam o programa, para propor melhorias e modificações, otimizando o alcance dos resultados esperados. Para tanto, foi pactuada, em Conferência de Consenso, a pergunta avaliativa: Como está implantada a assistência às mulheres durante o pós-parto na APS?

A realização de estudos de caso em municípios de diferentes contextos é uma opção metodológica plausível para a avaliação da implantação.

O modelo avaliativo poderá ser usado por gestores, profissionais, mulheres e demais interessados para elaborar políticas na área, compreender o funcionamento do programa, refletir sobre e qualificar a atenção pós-parto.

Tem o potencial para ser replicado, tanto em nível nacional quanto internacional, já que sua formulação considerou o contexto e operacionalização da atenção pós-parto com base em literatura/ documentos nacionais e internacionais, necessitando ajustes do modelo de acordo com a realidade. 


\section{Discussão}

$\mathrm{Na}$ ausência de uma teoria explícita e de uma organização claramente descrita da assistência pós-parto, foi fundamental desenvolver um modelo avaliativo para a sua compreensão e melhoria ${ }^{12}$. A construção do referido modelo está alinhada à concepção da Teoria da Mudança, que fornece uma descrição abrangente de como e por que uma mudança desejada se destina a acontecer, mapeando todas as etapas necessárias para atingir metas de longo prazo, e é uma ferramenta útil para acompanhar o progresso em direção a metas específicas 37.

O problema em questão é que o pós-parto é permeado por alterações psicológicas, físicas, sociais e econômicas $6,7,10,11,13,28$, que podem levar à morbimortalidade das mulheres $6,7,10,11,13,21,22,24$, sendo fundamental o cuidado pelos profissionais da APS, baseado nas necessidades e preferências das mulheres 13,20 .

A análise documental permitiu definir claramente o objetivo do programa, que foi ratificado pelos stakeholders, e apresentou coerência com as atividades desenvolvidas na assistência pós-parto. A convergência quanto aos objetivos do programa é primordial para a sua operacionalização lógica, o que leva ao alcance dos resultados esperados 16,18 .

A principal debilidade encontrada no programa foi a falta de definição de metas, tanto pelos stakeholders como pela literatura/documentos. As metas devem ser específicas, mensuráveis, alcançáveis e relevantes 18 e, portanto, sob esta perspectiva foram construídas - considerando as principais necessidades das mulheres - e pactuadas em Conferência de Consenso, podendo ser modificadas e/ ou ampliadas de acordo com cada realidade.

Para atingir as metas é necessário a compreensão sobre o programa 15,38. Assim, o MTA é um sistema hipotético-dedutivo que representa a teoria do objeto, explicitando como o mesmo idealmente funciona ${ }^{12}$. Sua construção demandou o entendimento de que a APS é a ordenadora do cuidado no âmbito da rede de atenção à saúde, deve atuar de forma articulada com outros pontos da rede e setores da sociedade 7,10,11,13,21,24,28, e atua junto às mulheres desde o pré-natal, com elaboração do plano pós-parto 1,20 .

No MTA, a delimitação dos contextos externo e político-institucional foi realizada com base na literatura/documentos e ratificada pelos stakeholders e especialistas, evidenciando que há uma compreensão clara sobre onde o programa está inserido e os aspectos que o envolvem, o que facilita sua implantação.

Para que ocorram as mudanças necessárias no programa, viabilizando atingir seus objetivos e metas, deve haver uma conexão entre atividades e resultados 15,38. Desse modo, o MLP apresentou a operacionalização da assistência pós-parto na APS, constituída por duas dimensões. A dimensão Gestão contempla aspectos essenciais para a efetivação do programa, já que demanda diversas ações no âmbito da organização do sistema de saúde municipal para que os profissionais da APS tenham condições de prestar assistência pós-parto de qualidade 7,11,13,21,22,24.

A dimensão Execução apresenta o rol de ações a serem desempenhadas no âmbito da APS para prestar assistência integral às mulheres no pós-parto.

Os documentos nacionais apresentaram fragilidade quanto à operacionalização da assistência pós-parto às mulheres. A Rede Cegonha 21,24, estratégia atual utilizada para a atenção obstétrica e neonatal, destaca poucas ações de cuidado para as mulheres, tendo como foco a criança. Recebe críticas pois considerando o próprio nome, "dessexualiza" a reprodução, dá ênfase ao concepto, desvincula a atenção ao parto e nascimento da Política Nacional de Assistência Integral à Saúde das Mulheres (PNAISM), e faz um reforço ao materno-infantilismo ao delimitar as prioridades 39 .

O protocolo de atenção básica para a saúde das mulheres 11 tem a perspectiva da atenção integral, porém descreve as atividades de forma genérica, sem especificar e detalhar com clareza como devem ser seguidas as recomendações, e não traz as evidências científicas que as embasaram. Assim, a fundamentação teórica utilizada para delimitar as ações na assistência pós-parto foi feita principalmente por meio da revisão sistemática de diretrizes clínicas internacionais 20 e revisão integrativa 13 .

Os stakeholders ratificaram o disposto pela literatura/documentos e foram essenciais para definir as ações quanto à violência doméstica e saúde sexual, já que são temas evidenciados pela literatura como importantes no pós-parto 29,31, porém pouco enfatizados em diretrizes clínicas, e no caso da saúde sexual há foco na contracepção 11,20. Os stakeholders deram ênfase à atenção à saúde mental, 
que é recomendada por diretrizes clínicas baseadas em alto nível de evidências científicas 20, porém negligenciada pelos profissionais da APS, especialmente no Brasil 13.

O envolvimento de múltiplos stakeholders é fundamental para definir a Teoria da Mudança do programa, já que os interessados podem ter opiniões diferentes sobre qual é a mudança desejada, por que ela é desejada e como pode e deve acontecer. Assim, o compartilhamento das visões melhora a definição do programa, minimiza conflitos e define papéis 38 .

Após desenvolver a teoria do programa por meio do Estudo de Avaliabilidade, a efetividade deste se dá por meio do seu uso, que é direcionado por intermédio das conclusões, recomendações e direcionamentos baseados nas recomendações 16. O presente Estudo de Avaliabilidade foi útil considerando-se que os stakeholders demonstraram interesse em compreender o programa e sua avaliação, e a Conferência de Consenso permitiu direcionar estas estratégias e definir a análise de implantação como uma possiblidade. Esse tipo de avaliação permite evidenciar o quanto o programa está operacionalizado, ou seja, as intervenções realizadas, déficits entre o planejado e o executado, além de permitir a análise das possíveis influências do contexto no grau de implantação, e do grau de implantação nos efeitos 12 .

O envolvimento de stakeholders com experiência prévia sobre o objeto no âmbito da gestão em saúde, assistência e participação social foi imprescindível para construir um modelo claro e ideal de assistência pós-parto. Por serem pessoas interessadas no programa, sua participação contribui para que o estudo seja executado plenamente, haja concordância com os critérios de avaliação, garantia de maior confiabilidade dos dados, aumento do uso da avaliação e do potencial de aprimoramento do programa desde o início do processo 18,19 .

Nessa direção, consideram-se os pressupostos da Teoria da Mudança, que implica o envolvimento das partes interessadas em todo o processo, viabilizando as mudanças necessárias para atingir as metas do programa 37,38 .

Os stakeholders foram essenciais para definir que a assistência pós-parto deve ocorrer de forma integral para mulheres com filhos vivos ou em situações de óbito fetal/infantil, considerando-se as necessidades específicas de cada mulher. Em geral, as diretrizes clínicas tratam do período pós-parto como uma fase crítica para mulheres e bebês, e direcionam as ações para o binômio mãe-filho, sem esclarecer sobre os cuidados às mulheres que não estejam com o recém-nascido 1,11,20.

Um Estudo de Avaliabilidade é uma ferramenta de tomada de decisão, por isto, é importante apresentar um conjunto claro de recomendações, com base nos objetivos da intervenção identificados por meio da Teoria da Mudança 15. Os resultados do Estudo de Avaliabilidade podem ser usados para direcionar um plano de avaliação, ou para determinar estratégias para facilitar a avaliabilidade, tal como ajustar os resultados do programa, modificar o programa, monitorar a implantação, ou providenciar assistência técnica para facilitar a melhoria de implementação em áreas específicas 16. Assim, neste trabalho, foram apresentadas diversas estratégias de possíveis usos do modelo avaliativo construído, que foi validado em conjunto com os stakeholders, potencializando o seu uso para as mudanças pretendidas 15 .

O modelo avaliativo construído não tem o intuito de negligenciar os cuidados aos bebês, mas sim dar ênfase às necessidades e cuidados pós-parto às mulheres, protagonizando-as, já que a atenção pós-parto, tanto nacional como internacional, ainda tem foco nos recém-nascidos com nulidade das mulheres 13,20. Com isso, pretende-se fomentar a mudança de foco da atenção pós-parto, da mulher reprodutora para a atenção integral às mulheres 38 .

Como limitações o estudo foi elaborado com base na demanda acadêmica, que pode apresentar menor uso quando comparado a estudos sob demanda institucional. Para superar esse aspecto, os stakeholders foram envolvidos em todo o processo de Estudo de Avaliabilidade, o que pode contribuir para o seu uso. Buscou-se envolver stakeholders da gestão, assistência e da sociedade civil, todos da Região Sul do Brasil por questões de operacionalização do trabalho, o que pode ter deixado de contemplar aspectos de outras realidades. Essa limitação foi minimizada pela ampla revisão da literatura e uso de documentos nacionais e internacionais, em que as contribuições dos stakeholders mantiveram alinhamento.

A principal potencialidade foi o uso de diferentes estratégias para garantir a validade e confiabilidade dos resultados, como o uso de referencial teórico adequado para um Estudo de Avaliabilidade, diferentes fontes de evidências (entrevistas, análise documental, revisão da literatura) e a Conferência de Consenso 14,19. 


\section{Considerações finais}

O estudo elaborou a teoria do programa, estabelecendo a contextualização e um desenho lógico do programa, com objetivo, metas, atividades, produtos, resultados e impacto, até então não sistematicamente explicitados na literatura e documentos sobre o tema, de acordo com a perspectiva dos stakeholders. Considerando sua compreensão e operacionalização lógica, foi possível determinar a avaliabilidade do programa.

O enfoque do Estudo de Avaliabilidade foi na formulação de um modelo orientado para a assistência integral à saúde das mulheres no pós-parto, tendo importante contribuição para fomentar a superação da visão materno-infantil, em geral, arraigada nos cuidados pós-parto.

A participação dos stakeholders em todo o Estudo de Avaliabilidade tem o potencial para ampliar seu conhecimento sobre o programa e mudar sua realidade. A teoria do programa definida por este trabalho pode ser utilizada por diversos atores, tanto em nível nacional quanto internacional, para implantar e/ou aprimorar a assistência pós-parto, implicando as mudanças necessárias para atingir as metas do programa.

\section{Colaboradores}

T. Baratieri e S. Natal participaram da concepção e projeto, análise e interpretação dos dados, redação, revisão crítica relevante do conteúdo intelectual e aprovação da versão final. Z. M. A. Hartz participou da análise e interpretação dos dados, revisão crítica relevante do conteúdo intelectual e aprovação da versão final. Todas as autoras são responsáveis por todos os aspectos do trabalho na garantia da exatidão e integridade de qualquer parte da obra.

\section{Informações adicionais}

ORCID: Tatiane Baratieri (0000-0002-0270-6395); Sonia Natal (0000-0001-6155-4785); Zulmira Maria de Araújo Hartz (0000-0001-9780-9428).

\section{Agradecimentos}

Agradecemos aos stakeholders e especialistas que participaram da pesquisa, disponibilizando seu tempo em todas as etapas do estudo e compartilhando o seu conhecimento. 


\section{Referências}

1. ACOG Committee Opinion No. 736: optimizing postpartum care. Obstet Gynecol 2018; 131:e140-50.

2. Wilkie S, Crawley R, Button S, Thornton A, Ayers S. Assessing physical symptoms during the postpartum period: reliability and validity of the primary health questionnaire somatic symptom subscale (PHQ-15). J Psychosom Obstet Gynaecol 2018; 39:56-63.

3. Ayers S, Crawley R, Button S, Thornton A, Field AP, Flood C, et al. Evaluation of expressive writing for postpartum health: a randomised controlled trial. J Behav Med 2018; 41:614-26.

4. Langlois ÉV, Miszkurka M, Zunzunegui MV, Ghaffar A, Ziegler D, Karp I. Inequities in postnatal care in low- and middle-income countries: a systematic review and meta-analysis. Bull World Health Organ 2015; 93:25970.

5. Kleppel L, Suplee PD, Stuebe AM, Bingham D. National initiatives to improve systems for postpartum care. Matern Child Health J 2016; 20:66-70.

6. National Institute for Health and Care Excellence. Postnatal care up to 8 weeks after birth. London: National Institute for Health and Care Excellence; 2015.

7. World Health Organization. WHO recommendations on postnatal care of the mother and newborn. Geneva: World Health Organization; 2013.

8. Robling M, Bekkers MJ, Bell K, Butler CC, Cannings-John R, Channon S, et al. Effectiveness of a nurse-led intensive home-visitation programme for first-time teenage mothers (Building Blocks): a pragmatic randomised controlled trial. Lancet 2016; 387:146-55.

9. Brodribb W, Zadoroznyj M, Nesic M, Kruske S, Miller YD. Beyond the hospital door: a retrospective, cohort study of associations between birthing in the public or private sector and women's postpartum care. BMC Health Serv Res 2015; 15:14.

10. Grupo de Trabajo de la Guía de Práctica Clínica de Atención en el Embarazo y Puerperio. Guía de práctica clínica de atención en el embarazo y puerperio. s.l.: Ministerio de Sanidad, Servicios Sociales e Igualdad/Agencia de Evaluación de Tecnologías Sanitarias de Andalucía; 2014.

11. Ministério da Saúde; Instituto Sírio-Libanês de Ensino e Pesquisa. Protocolos da atenção básica: saúde das mulheres. Brasília: Ministério da Saúde; 2016.

12. Hartz ZMA, Silva LMV, organizadoras. Avaliação em saúde: dos modelos teóricos à prática na avaliação de programas e sistemas de saúde. Rio de Janeiro: Editora Fiocruz; 2005.

13. Baratieri T, Natal S. Ações do programa de puerpério na atenção primária: uma revisão integrativa. Ciênc Saúde Colet 2019; 24:422738.
14. Baratieri T, Nicolotti CA, Natal S, Lacerda JT. Aplicação do estudo de avaliabilidade na área da saúde: uma revisão integrativa. Saúde Debate 2019; 43:231-46.

15. Craig P, Campbell M. Evaluability assessment: a systematic approach to deciding whether and how to evaluate programmes and policies. http://whatworksscotland.ac.uk/wp-content/ uploads/2015/07/WWS-Evaluability-Assess ment-Working-paper-final-June-2015.pdf (acessado em 20/Jan/2018).

16. Trevisan MS, Walser TM. Evaluability assessment: improving evaluation quality and use. Thousand Oaks: SAGE Publications; 2015.

17. Leviton LC, Khan LK, Rog D, Dawkins N, Cotton D. Evaluability assessment to improve public health policies, programs, and practices. Annu Rev Public Health 2010; 31:213-33.

18. Thurston WE, Ramaliu A. Evaluability assessment of a survivors of torture program: lessons learned. Can J Program Eval 2005; 20:125.

19. Walser TM, Trevisan MS. Evaluability assessment thesis and dissertation studies in graduate professional degree programs: review and recommendations. Am J Eval 2016; 37:118-38.

20. Baratieri T, Soares LG, Kappel EP, Natal S, Lacerda JT. Recomendações para o cuidado pós-parto às mulheres na atenção primária: revisão sistemática. Rev APS 2020; no prelo.

21. Ministério da Saúde. Portaria no 1.459, de 24 de junho de 2011. Institui, no âmbito do Sistema Único de Saúde - SUS - a Rede Cegonha. Diário Oficial da União 2011; 28 jun.

22. Departamento de Atenção Básica, Secretaria de Atenção à Saúde, Ministério da Saúde. Atenção ao pré-natal de baixo risco. Brasília: Editora do Ministério da Saúde; 2012. (Cadernos de Atenção Básica, 32).

23. Ministério da Saúde. Cadernos HumanizaSUS: humanização do parto e do nascimento. Brasília: Ministério da Saúde; 2014.

24. Ministério da Saúde. Portaria no 650 , de 5 de outubro de 2011. Dispor sobre os Planos de Ação regional e municipal da Rede Cegonha. Diário Oficial da União 2011; 5 out.

25. Bezerra LCA, Cazarin G, Alves CKAA. Modelagem de programas: da teoria à operacionalização. In: Samico I, Felisberto E, Figueiró AC, Frias PG, organizadores. Avaliação em saúde: bases conceituais e operacionais. Rio de Janeiro: MedBook; 2010. p. 65-78.

26. Souza LEPF, Silva LMV, Hartz ZMA. Conferência de consenso sobre a imagem-objetivo da descentralização da atenção à saúde no Brasil. In: Hartz ZMA, Silva LMV, organizadoras. Avaliação em saúde: dos modelos teóricos à prática na avaliação de programas e sistemas de saúde. Rio de Janeiro: Editora Fiocruz; 2005. p. 65-102.

27. Miles MB, Huberman AM, Saldaña J. Qualitative data analysis: a methods sourcebook. 3 a Ed. Thousand Oaks: SAGE Publications/Arizona State University; 2014. 
28. National Collaborating Centre for Primary Care. Postnatal care: routine postnatal care of women and their babies. London: Royal College of General Practitioners; 2006.

29. McDonald E, Woolhouse H, Brown SJ. Consultation about sexual health issues in the year after childbirth: a cohort study. Birth 2015; 42:354-61.

30. Giallo R, Pilkington P, McDonald E, Gartland D, Woolhouse H, Brown S. Physical, sexual and social health factors associated with the trajectories of maternal depressive symptoms from pregnancy to 4 years postpartum. Soc Psychiatry Psychiatr Epidemiol 2017; 52:815-28.

31. Moraes CL, Oliveira AGS, Reichenheim ME, Gama SGN, Leal MC. Prevalência de violência física entre parceiros íntimos nos primeiros seis meses após o parto no Município do Rio de Janeiro, Brasil. Cad Saúde Pública 2017; 33:e00141116.

32. Lobato G, Moraes CL, Reichenheim ME. Magnitude da depressão pós-parto no Brasil: uma revisão sistemática. Rev Bras Saúde Mater Infant 2011; 11:369-79.

33. Albuquerque APS, Pitangui ACR, Rodrigues PMG, Araújo RC. Prevalência da gravidez de repetição rápida e fatores associados em adolescentes de Caruaru, Pernambuco. Rev Bras Saúde Mater Infant 2017; 17:355-63.
34. World Health Organization. Global targets 2025. https://www.who.int/nutrition/globaltarget-2025/en/ (acessado em 26/Fev/2019).

35. Faculty of Sexual and Reproductive Health. Contraception after pregnancy. https://www. fsrh.org/news/new-fsrh-guideline--contra ception-after-pregnancy/ (acessado em 04/ Nov/2017).

36. National Institute for Health and Care Excellence. Antenatal and postnatal mental health: clinical management and service guidance. https://www.nice.org.uk/guidance/cg 192 (acessado em 04/Nov/2017).

37. Vogel I. Review of the use of "Theory of Change" in international development: review report. https://isabelvogel.co.uk/ (acessado em 23/Abr/2019).

38. van Es M, Guijt I, Vogel I. Theory of change thinking in practice: a stepwise approach. Den Haag: Hivos; 2015. (Hivos ToC Guidelines).

39. Aquino EML. Para reinventar o parto e o nascimento no Brasil: de volta ao futuro. Cad Saúde Pública 2014; 30 Suppl 1:S8-10. 


\section{Abstract}

Postpartum care for women in primary healthcare (PHC) is important for reducing their morbimortality, but there is no clearly described theory on such care, and the assessment studies are rare. This study aimed to develop and systematize an assessment model for women's postpartum care in PHC, verifying it evaluability. An evaluability study was performed using a Brazilian and international literature review, review of national documents, and interviews with stakeholders. Such evidence backed the elaboration of an assessment model that was validated in a consensus workshop. The data were analyzed with thematic analysis. The study elaborated the program's theory, in which postpartum care in PHC ideally takes place with a comprehensive approach to the woman's physical, psychological, emotional, and social needs, considering the individuality of women with liveborn children or in situations of fetal/neonatal death, initiating prenatal care and continuity in the postpartum period, and with the involvement of spouses and other family members. The program's theory established the program's contextualization and logical design, with objectives, targets, activities, outputs, results, and impact, previously not explained systematically in the literature and documents. Postpartum care was found to be evaluable through an implementation analysis, and the program's theory was defined, with the potential for use by various stakeholders at both the national and international levels to implement and/or improve comprehensive postpartum care for women.

Postpartum Period; Health Evaluation; Women's Health; Primary Health Care

\section{Resumen}

La asistencia posparto a mujeres en la atención primaria de salud (APS) es importante para la reducción de la morbimortalidad entre ellas, no obstante, no existe una teoría de esa asistencia claramente descrita y los estudios evaluativos son escasos. El objetivo de este estudio fue desarrollar y sistematizar un modelo evaluativo de la asistencia a mujeres durante el posparto en la APS, verificando su evaluabilidad. Se realizó un estudio de evaluabilidad mediante la revisión de la literatura nacional e internacional, revisión de documentos nacionales y entrevistas con grupos de interés. Tales evidencias fundamentaron la elaboración del modelo evaluativo que se validó en una conferencia de consenso. Se utilizó un análisis temático para el análisis de datos. El estudio elaboró la teoría del programa, donde la asistencia posparto en la APS necesita producirse idealmente de forma integral, conforme las necesidades de salud física, psicológica, emocional y social; considerar la individualidad de las mujeres con hijos vivos o en situaciones de óbito fetal/infantil; iniciar el cuidado prenatal $y$ tener continuidad en el posparto, con implicación de las familias/compañeros(as). La teoría del programa estableció la contextualización y un diseño lógico del programa, con objetivos, metas, actividades, productos, resultados e impacto, hasta entonces no sistemáticamente explicitados en la literatura y documentos. Se verificó que la asistencia posparto está disponible mediante un análisis de implantación y que la teoría del programa definido puede utilizarse por parte de diversos actores, tanto a nivel nacional, como internacional, para implantar $y /$ perfeccionar la asistencia integral a las mujeres en el posparto.

Periodo Posparto; Evaluación en Salud; Salud de la Mujer; Atención Primaria de Salud
Recebido em 08/Mai/2019

Versão final reapresentada em 17/Dez/2019 Aprovado em 30/Dez/2019 